\title{
Lexis
}

Journal in English Lexicology

HS 1 | 2009

Lexicology \& Phonology

\section{Graphie, phonie et encodage dialectal : le Cockney de Somerset Maugham dans Liza of Lambeth}

\section{Manuel Jobert}

\section{(2) OpenEdition}

\section{Journals}

\section{Édition électronique}

URL : http://journals.openedition.org/lexis/905

DOI : 10.4000/lexis.905

ISSN : 1951-6215

Éditeur

Université Jean Moulin - Lyon 3

\section{Référence électronique}

Manuel Jobert, « Graphie, phonie et encodage dialectal : le Cockney de Somerset Maugham dans Liza of Lambeth », Lexis [En ligne], HS 1 | 2009, mis en ligne le 17 septembre 2009, consulté le 19 avril 2019. URL : http://journals.openedition.org/lexis/905 ; DOI : 10.4000/lexis.905

\section{(c) (i) ()}

Lexis is licensed under a Creative Commons Attribution-NonCommercial-NoDerivatives 4.0 International License. 


\title{
Graphie, phonie et encodage dialectal : le Cockney de Somerset Maugham dans Liza of Lambeth
}

Manuel Jobert ${ }^{1}$

\section{Résumé}

L'objet de cet article est d'établir un lien entre l'écriture phonétique (pronunciation spelling) et encodage dialectal. On se rend compte en effet que l'on retrouve en littérature des fonctionnements déjà présents en langue. En se fondant sur Liza of Lambeth, il apparaît que trois manipulations grapho-phonologiques (ajout, effacement, substitution) seulement sont nécessaires pour encoder le dialecte Cockney.

Mots-clés :

Encodage dialectal - écriture phonétique - phonostyle - code orthographique

$* * *$

\begin{abstract}
The purpose of this paper is to show the similarity between pronunciation spelling and dialect encoding. It appears that the grapho-phonological manipulations present in fictional texts also exist in the English language. In Somerset Maugham's Liza of Lambeth, only three types of dialect encoding (grapheme insertion, suppression and substitution) are sufficient to encode the Cockney dialect.
\end{abstract}

\section{Key-words:}

Dialect encoding - pronunciation spelling - phonostyle - institutional spelling

\footnotetext{
${ }^{1}$ Université Jean Moulin - Lyon 3, CREA - EA 370 : manuel.jobert@univ-lyon3.fr
} 


\section{Introduction}

L'encodage du dialecte dans la fiction est avant tout une manipulation graphique dont l'objet est de représenter un accent / dialecte cible de manière plus efficace que l'orthographe institutionnalisée. L'écart entre l'orthographe traditionnelle et l'encodage peut être plus ou moins marqué, allant de la touche impressionniste à un encodage quasi systématique. L'encodage systématique nécessite le recours à un alphabet phonétique qui établit un rapport de bi-univocité entre le phonème et le graphème ${ }^{2}$. On sait que la lecture ne suppose pas une reconnaissance individuelle de toutes les lettres. Le test de Reicher prouve qu'il est plus aisé de reconnaître une lettre dans une séquence graphique que lorsqu'elle est isolée. Dehaene [2007 : 81] explique :

Nous ne pouvons pas reconnaître une lettre seule sans bénéficier immédiatement du contexte dans lequel elle est présentée. Ce contexte, s'il forme un mot ou un fragment de mot, donne accès à des niveaux supplémentaires de codage (graphèmes, syllabes, morphèmes) dont les "votes » s'ajoutent à ceux des lettres et en facilitent la perception. La plupart de modèles de lecture expliquent l'effet Reicher en supposant que les interactions entre lettres et mots fonctionnent dans les deux sens : les unités de niveau supérieur conspirent pour favoriser la détection des lettres qui sont compatibles avec leur propre interprétation. Ainsi, ce que nous voyons dépend de ce que nous croyons voir.

Ainsi, l'encodage dialectal n'est généralement pas un obstacle à la lecture même quand on ne connaît pas l'accent cible. Le processus de lecture est suffisamment sophistiqué pour compenser certaines idiosyncrasies graphiques. Dans "Only the Dead Know Brooklyn ", Thomas Wolfe [Ahmad 2007: 283] propose un exemple d'encodage du Brooklynese, le basilecte new-yorkais. On peut, avant de présenter la version de l'auteur, se livrer à un test de lecture en supprimant les graphèmes modifiés :

_ere's no guy livin__ at knows Brooklyn t_o_an' t_ro_ (only the dead know Brooklyn t_ro_and t_ro_).

En contexte, la lecture par voie lexicale permet de reconstruire :

There's no guy living that knows Brooklyn through and through (only the dead know Brooklyn through and through).

L'encodage dialectal opéré par l'auteur ne pose pas plus de problème, voire même un peu moins, étant donné que certaines manipulations s'appuient sur un fonctionnement plus régulier de la langue. Au niveau de la conversion des graphèmes en phonèmes, plus les graphèmes s'écartent de la prononciation des lettres isolées (plus ils sont irréguliers), plus le temps de traitement est long. Si le lecteur parvient à décoder instantanément l'encodage dialectal, c'est qu'il utilise simultanément les deux voies de lecture (voie lexicale et voie phonologique). En d'autres termes, «des informations sur la prononciation des mots sont automatiquement activées » [Dehaene 2007 : 53], même si le lecteur n'en a pas conscience. La prononciation régulière des graphèmes, si elle fait sens, sera retenue.

Dere's no guy livin' dat knows Brooklyn t'roo an' t'roo (only the dead know Brooklyn t'roo and t'roo).

\footnotetext{
${ }^{2}$ A l'origine, l'Alphabet Phonétique International avait pour vocation de se substituer à l'orthographe, au moins dans l'enseignement des langues étrangères.
} 
On remarque dans cet exemple plusieurs cas de TH-fronting $(/ \theta /$ et $/ ð / \rightarrow / \mathrm{t} /$ et $/ \mathrm{d} /)$ pour there, that, through. Au niveau graphique, on passe alors de $<\mathrm{th}>$ à $<\mathrm{t}>$ ou à $<\mathrm{d}>$. On note néanmoins que the dead n'est pas modifié. La forme livin' indique que le $/ \mathrm{n} / \mathrm{n}$ 'est pas vélarisé, ce qui fait rimer living avec Brooklyn. Enfin, le digraphe $<00>$ est substitué à $<$ ough> dans through, ce qui constitue une régularisation de l'orthographe, plutôt que la notation d'un accent particulier. Il faut donc distinguer les manipulations orthographiques qui changent la prononciation en évoquant un accent particulier, des autres, qui marquent l'oralité. Les deux procédés peuvent d'ailleurs coexister à l'intérieur d'une même séquence graphique (t'roo). Enfin, il n'est pas nécessaire que l'encodage soit systématique (Dere / the) pour que les informations nécessaires parviennent au lecteur.

Traditionnellement, l'encodage dialectal dans la fiction intéresse plus le littéraire que le linguiste et l'on étudie plus volontiers les prolongements esthétiques ou interprétatifs du procédé que son mécanisme. Toutefois, le procédé utilisé est comparable à l'écriture phonétique (pronunciation spelling). L'encodage du dialecte en fiction sera appréhendé comme une extension stylistique de l'écriture phonétique. Si cette extension est possible, c'est qu'elle se fonde sur un fonctionnement grapho-phonologique identique en langue.

Ainsi interrogées, les relations entre graphie et phonie permettent d'appréhender de manière indirecte le lien qui unit ou sépare phonologie et lexicologie / lexicographie. On pourrait objecter qu'il existe un écart entre graphie et lexicologie. On sait toutefois que certains cas d'écriture phonétique intègrent le lexique comme par exemple le mot hiccup, ou font leur apparition dans la «zone floue » dont parle Tournier [1993], comme wanna et gonna. On considère qu'un mot intègre le lexique d'une langue quand il entre dans le lexique mental (mental lexicon) de ses locuteurs. Mais il est impossible de savoir ce qui est dans le lexique mental de tous les locuteurs d'une langue. L'intégration au lexique est donc corrélée au figement orthographique d'une part et à l'entrée dans un ou plusieurs dictionnaires d'autre part.

On s'intéressera à l'accent / dialecte Cockney, probablement l'un des plus encodés dans la fiction anglaise, et Liza of Lambeth, de Somerset Maugham, servira de corpus principal à cette étude. Avant cela, il convient de s'interroger sur les relations qui unissent prononciation et orthographe dans la langue anglaise.

\section{L'écriture phonétique ou pronunciation spelling}

Saussure [1916:45], explique la relation entre l'oral et l'écrit ainsi :

Langue et écriture sont deux systèmes de signes distincts ; l'unique raison d'être du second est de représenter le premier; l'objet linguistique n'est pas défini par la combinaison du mot écrit et du mot parlé ; ce dernier constitue à lui seul cet objet. Mais le mot écrit se mêle si intimement au mot parlé dont il est l'image, qu'il finit par usurper le rôle principal ; on en vient à donner autant et plus d'importance à la représentation du signe vocal qu'à ce signe lui-même. C'est comme si l'on croyait que, pour connaître quelqu'un, il vaut mieux regarder sa photographie que son visage.

L'importance de l'écrit dans nos cultures tend à faire oublier ce principe et donne une légitimité plus grande à la forme écrite. La langue anglaise, en raison de son histoire contrariée, a subi des modifications considérables au cours des siècles, tant sur les plans orthographique que phonétique. En effet, la plus latine des langues germaniques porte aujourd'hui encore la marque des tensions souvent contradictoires qui l'animent ${ }^{3}$. L'écriture

\footnotetext{
${ }^{3}$ Sur tous ces points, voir McIntyre [2009].
} 
phonétique peut être définie comme un ajustement du code graphique à la prononciation d'un mot.

\subsection{L'écriture phonétique institutionnalisée}

L'évolution de la langue a conduit à la modification de certaines formes orthographiques qui sont maintenant reconnues par l'institution. Ainsi, les digraphes $<$ oe $><$ ae $>$ ont été simplifiés en $<\mathrm{e}>$ dans economy ( $<$ latin oeconomia), premium $(<$ latin præmium), ou encore enigma ( $<$ latin ænigma). Seule l'orthographe $<\mathrm{e}>$ est aujourd'hui possible. Dans d'autres cas, anaemia / anemia, anaesthesia / anesthesia, gynaecology / gynecology, oesophagus / esophagus, paediatrician / pedriatician, encyclopaedia / encyclopedia, etc. les deux orthographes coexistent. Il y a donc une continuité dans la simplification de ces mots avec une tendance de plus en plus grande à utiliser l'orthographe simplifiée $<\mathrm{e}>$.

On doit à Noah Webster la simplification orthographique de l'anglais américain. Ces modifications rendent la graphie plus proche la prononciation. On notera par exemple la terminaison -or pour remplacer -our avec colo $(u) r$, hono $(u) r$, favo(u)rite ${ }^{4}$. La plupart des mots se terminant en -our avaient une origine latine en -or. Après 1066, la tendance fut d'adopter la graphie - our pour se rapprocher de l'orthographe française. Par ailleurs, les mots qui se terminent en -bre / -tre comme theatre, litre, centre, fibre en anglais britannique donnent $-e r$ en anglais américain. En revanche, les mots en -cre conservent l'ordre $<$ re $>$ en anglais américain pour indiquer que la prononciation est $/ \mathrm{k} /$.

D'autres cas de réajustement, moins systématiques, existent et permettent de distinguer sans ambigüité l'anglais britannique de l'anglais américain :

\begin{tabular}{|c|c|}
\hline anglais britannique & anglais américain \\
\hline dialogue & dialog \\
programme & program \\
tonne & ton \\
annexe & annex \\
cosy & cozy \\
kerb & curb \\
plough & plow \\
sceptic & skeptic \\
tyre & tire \\
etc. & \\
\hline
\end{tabular}

Certains cas d'orthographe phonétique concernent des mots isolés. L'écriture du mot /'hrk $\Lambda \mathrm{p} /$ fut à l'origine, et contrairement à son étymologie (< français hoquet, danois, hikke), orthographié hiccough (1626) par analogie avec cough. L'écriture hiccup date de 1788. Le mot a donc subi une modification orthographique afin de le mettre en conformité avec sa prononciation et son étymologie. L'Oxford Dictionary of English et le Webster ne proposent que l'entrée hiccup et notent la variante orthographique hiccough. Le LPD 2008 fait l'inverse, ce qui indique que le statut orthographique de ce mot n'est pas tout à fait tranché. Le mot /'dzerl/ est quant à lui intéressant dans la mesure où les deux formes orthographiques jail / gaol coexistent, la forme américaine (jail) représentant un cas de réécriture phonétique. On

\footnotetext{
${ }^{4}$ Toutefois glamour maintient le $<\mathrm{u}>$ en raison de son origine écossaise.
} 
pense également à /'ba:kli/ pour Barclay et Berkeley alors que l'américain prononce le second /'b3:rkli/.

Une analyse synthétique de l'évolution de l'orthographe de la langue anglaise est impossible ici. On sait qu'il fallut attendre la fin du XIXe siècle pour parvenir à un semblant de consensus en la matière ${ }^{5}$. Et encore, il suffit de lire des romans du XIXe siècle pour se rendre compte de l'instabilité orthographique (on trouve par exemple chuse chez Jane Austen). Dans tous les cas, on constate que les modifications orthographiques tendent à rendre la forme écrite plus conforme à sa prononciation, dans la limite toutefois des possibilités graphiques. Ainsi, l'orthographe américaine $<$ plow $>$ règle la question de la prononciation du digraphe $<\mathrm{gh}>$, en l'effaçant, mais laisse planer le doute sur la réalisation $\mathrm{du}$ digraphe $<\mathrm{ow}>$, généralement prononcé /av/ mais dont la réalisation concurrente est /əv/, comme le prouvent les paires minimales du type row/row, bow/bow, Moscow (GB) / Moscow (US) ou encore David Bowie prononcé /əv/ par les Britanniques et /av/par les Américains.

A côté de ces formes dûment attestées, existent des formes intermédiaires du type fish ' $n$ ' chips ou Rock ' $n$ ' Roll, dont l'orthographe phonétique (la forme <' $\mathrm{n}>$ pour noter la forme faible de and) est plus fluctuante. Le LPD par exemple propose l'entrée Rock ' $n$ ' Roll alors que le EPD, de manière un peu surprenante, écrit Rock-and-Roll. Cela est une preuve supplémentaire que l'institution n'intègre que très lentement les innovations orthographiques.

\subsection{L'écriture phonétique de la marge}

D'autres cas d'écriture phonétique se trouvent dans le langage publicitaire, la bande dessinée ou les SMS par exemple. Ces cas constituent des manipulations graphiques à des fins stylistiques. De nombreuses boissons ou produits alimentaires, comme la bière Miller, utilisent volontiers l'adjectif lite. Ce cas classique est fondé sur le fonctionnement régulier de la langue.

$<\mathrm{i}>\rightarrow / \mathrm{aI} / /_{-} \mathrm{CV \#}$

Le graphème $-g h$ comme dans bright, night, fight est, au cours du temps, devenu silencieux, provoquant l'allongement de la voyelle : (knight : knIxt $\rightarrow$ nart). Dans cette publicité, lite est traité comme bite, nice, site, rejoignant ainsi l'orthographe nite pour night (attestée dès 1931). Ce type de simplification est fondé sur le fonctionnement régulier de la langue, ce qui permet un décodage aisé. La ressemblance avec le cas night / nite montre bien qu'il ne s'agit pas d'un phénomène nouveau.

Les caricaturistes manipulent volontiers la graphie pour représenter la chaîne parlée de certains locuteurs. Le caricaturiste Steve Bell ${ }^{6}$ du Guardian publiait en 2008, un dessin de George W. Bush avec le texte suivant, censé représenter la parlure du Président :

\section{John McCain is my kinda outcast.}

Cela rend compte de la contraction de kind of traditionnellement encodée kinda. On retrouve, sous cette même stratégie, les termes wanna, gonna, watcha, outa. Wanna et gonna sont d'ailleurs des entrées distinctes du LPD 2008, ce qui souligne leur intégration dans la langue. On a affaire ici à des cas de troncation + amalgame souvent associés à l'anglais américain :

\footnotetext{
${ }^{5}$ On pense ici à l'entreprise de Swift, voir McIntyre [2009: 155].

${ }^{6}$ Les caricatures de Steve Bell sont disponibles sur le site : http://www.guardian.co.uk/
} 


$\begin{array}{ll}\text { Kind of } & \rightarrow \text { kinda } \\ \text { Want to } & \rightarrow \text { wanna } \\ \text { Going to } & \rightarrow \text { gonna } \\ \text { What do you } & \rightarrow \text { Watcha } \\ \text { Out of } & \rightarrow \text { outa }\end{array}$

Si ces graphies évoquent les formes oralisées avec plus d'efficacité que l'orthographe traditionnelle, on remarque néanmoins une représentation parfois partielle de la réalité phonétique.

Si of peut être réduit à un schwa dans kind of cute, wanna pose plus de problèmes. On sait qu'en anglais américain, l'enchaînement de la séquence $n t$ peut donner lieu à un effacement $\mathrm{du} / \mathrm{t} /$ dans une langue familière (center $\rightarrow$ /'sentər/, l'italique indiquant l'élision optionnelle $\mathrm{du} / \mathrm{t} /$ ). La même élision peut apparaître en anglais britannique familier. La graphie $<\mathrm{a}>$, en revanche, ne rend que très mal compte de la prononciation $/ \mathfrak{w} /$ de l'anglais britannique puisqu'elle implique la conservation de la règle phonologique voulant que $<\mathrm{a}>$ précédée d'un son $/ \mathrm{w} / \mathrm{s}$ 'arrondisse en $/ \mathrm{p} /$. Une graphie plus proche de la réalité serait *wonna, comme dans gonna. Mais Wells note dans LPD 2008, que la prononciation britannique de gonna est /gənə/. Les prononciations du type /gnnə/ seraient en fait des prononciations orthographiques d'orthographe phonétique wanna... Ces formes, même si elles se rapprochent de la réalité phonétique, ne parviennent toutefois pas à la refléter fidèlement.

Les modifications écrites sont parfois utilisées pour encoder un accent particulier. Steve Bell en 2008 mettait en scène Tony Blair avec un membre de l'aristocratie anglaise qui lui dit :

\section{Mr Blah. A Friendly word of advice. Fack orfff and take your ill-bred slut of a} wife with you.

L'orthographe Blah pour Blair et Fack orfff pour fuck off évoque les spécificités des voyelles de la conservative $R P$ ou $U-R P$ (Wells [1982]). On remarque ici que la graphie Blah pour Blair indique que la diphtongue de SQUARE (/eə/) se termine de manière très ouverte. La graphie orff pour off, renvoie à la tendance de la $U-R P$ d'avoir /o:/ devant une fricative sourde alors que la Mainstream $R P$ adopte / $\mathrm{p} /$. Cette présentation humoristique est instantanément décodée par le lecteur anglais qui, en appliquant les règles de lecture de sa langue, reproduit, avec plus ou moins de précision, les traits stéréotypiques de l'aristocratie. Il convient de noter qu'un lecteur américain, ou britannique s'il a un accent rhotique, pourra mal décoder la présence $\mathrm{du}<\mathrm{r}>$ dans orfff, qui n'indique pas la prononciation $\mathrm{du}<\mathrm{r}>$ mais bien l'allongement de la voyelle qui précède.

Il en va de même pour l'idiolecte de George Bush qui, s'adressant au géant géorgien, dit :

\section{Do as I say or be froze outa the irranational commudiny.}

Plusieurs encodages sont effectués. Le problème grammatical ainsi que l'inversion des deux dernières syllabes de community renvoient à une mauvaise maîtrise de la langue de la part du président. A noter que Communidy rend compte graphiquement de la battue alvéolaire voisée américaine : /kə'mju:nəti/. La forme outa est comparable aux exemples déjà vus. Reste la forme irranational qui elle aussi renvoie à la battue alvéolaire en contexte relâché et avec compression, associée à une inversion de syllabes. Le jeu de mots (au moins visuel) international / irrational doit, quant à lui, être attribué au caricaturiste. 
Ces derniers exemples, appartenant à un genre particulier, ne sont efficaces que parce qu'ils sont décodés instantanément pas le lecteur. Il s'agit d'un effet de style mais le succès des formes wanna et gonna, montre que si une forme est reprise de manière systématique, elle a une chance d'être intégrée dans le lexique institutionnel. Dans le même ordre d'idées, on trouve le mot Strine, forme tronquée souvent utilisée pour parler de l'accent australien (caractérisé par ses diphtongues proches du Cockney : le /ei/ de Australian devient /ai/ dans Strine), le mot thru', forme archaïque mais aujourd'hui utilisée dans des contextes informels, ou encore l'orthographe fella, pour fellow.

\subsection{Texto et Internet}

Le textese et le $e$-discourse ou Netspeak méritent aussi d'être évoqués ici en raison de leur fréquence d'utilisation. Dans un article intitulé « $2 \mathrm{~b}$ or not $2 \mathrm{~b}$ » (The Guardian Weekly, 25.07.08), David Crystal analyse le fonctionnement du textese. Il défend que la siglaison du type CMB (call me back) ou LOL (laughing out loud), aujourd'hui le titre d'un film, relèvent des mêmes manipulations que le IOU qui date de 1618 ou le SWALK (sealed with a loving kiss) que les générations précédentes utilisaient dans leur courrier amoureux. Plus près de notre problématique, des exemples de messages du type 'cos ou wot ne sont en fait pas des inventions récentes mais sont répertoriées dans le OED depuis 1828 pour le premier et 1829 pour le second. Crystal donne l'exemple suivant et commente :

iowan $2 b w u$

[I only want to be with you].

On y trouve :

- l'utilisation du mot entier $I$

- l'initialisation $o$

- la troncation wan

- le logogramme 2 (signe unique qui représente un mot complet)

- le logogramme $b$

- l'initialisation $w$

- le logogramme $u$

Ce sont donc des procédés linguistiques complexes qui sont mis en œuvre et, pour la plupart, des procédés anciens. Cette capacité de la langue à créer et à modifier rend bien compte de la tension entre la graphie d'un mot et sa phonie, ou de manière plus générale, entre sa morphologie et sa phonologie. A cette malléabilité de la langue correspond la grande adaptabilité du lecteur / décodeur. Depuis que l'écrit a été utilisé pour représenter l'oral, des formes non institutionnelles ont été inventées pour que le rapport entre orthographe et prononciation soit plus étroit. De même qu'une langue peut être définie comme un dialecte qui a bien tourné, une forme écrite novatrice peut entrer dans la langue. Comme le souligne David Crystal, nombre de ces formes appartiennent à une tradition littéraire. Stockwell [2007] donne quelques exemples tirés de blogs ou de forums de discussion. On y retrouve puter pour computer, qui rappelle fridge pour refridgerator ainsi que des formes semblables au textese ( $u$ pour you, ur pour your). Certaines formes graphiques évoquent aussi des prononciations particulières, ainsi stoopid pour stupid (élision $\mathrm{du} / \mathrm{j} /$ ) ou somethin' (forme non vélarisée de la forme $-\mathrm{ING}$ ) pour something. On serait tenté de voir dans ces formes un point de contact entre l'écrit et l'oral. Stockwell [2007 : 119] commente : 
Neither the normal patterns of writing nor the characteristic features of speech apply perfectly to this mode of discourse, leading [Chadwick \& Holdstock] to the conclusion that email, electronic chat and telephone texting all represent not a 'mid-point' between speech and writing but a new, third mode of discourse.

Il y a en effet dans ce type de discours d'autres phénomènes que ceux relevant de l'écriture phonétique (les émoticons pas exemples :) !). Il n'en reste pas moins que l'on peut parler d'un véritable retour en force de l'écriture phonétique par ce biais technologique. Les procédés utilisés n'ont rien de nouveau mais l'exposition de toutes les couches de la population à cette nouvelle écriture constitue un virage décisif dans les liens qui unissent l'écrit et l'oral.

L'écriture phonétique est aussi particulièrement présente, depuis toujours, dans le texte de fiction, sous la plume d'auteurs qui souhaitent retranscrire l'accent ou le dialecte de leurs personnages.

\section{L'encodage du dialecte dans la fiction}

\subsection{Réalisme et effet de réel}

Le statut du dialecte dans la fiction a donné lieu à de nombreuses études auxquelles on ne peut que renvoyer, par exemple Chapman [1994] pour la littérature britannique et Jones [1999] pour la littérature américaine. S'il n'est pas question de reprendre les conclusions de ces auteurs, il convient néanmoins de se livrer à un rapide état des lieux afin de circonscrire les enjeux.

$\mathrm{Au}$ Royaume-Uni, l'époque victorienne fut particulièrement riche en termes d'encodage dialectal mais on sait que, de Chaucer (voir The Reeve's tale pour l'accent du nord) à Irvine Welsh (pour le Scots moderne), le phénomène a toujours existé, même si les motivations des auteurs sont différentes. Aux Etats-Unis, le Gilded Age donna lieu à un regain d'intérêt pour le dialecte littéraire, the cult of the vernacular pour reprendre Jones [1999: i]. Certains auteurs comme Dickens, Hardy, Gaskell, Emily Brontë, Scott, George Washington Cable, Chopin, Twain, Crane, etc. ont fait l'objet d'études spécifiques des dialectes qu'ils utilisent. On se reportera par exemple à Rotten English, une anthologie qui réunit des textes particulièrement riches en encodage dialectal.

Comme le rappelle Ferguson [1998], la critique s'est principalement intéressée à deux aspects de l'encodage dialectal : la cohérence (un personnage qui parle un dialecte le parle-t-il de manière cohérente ?) et la précision (l'encodage est-il fidèle au dialecte qu'il est censé représenter ?). Or, la graphie a ses limites et la précision de l'encodage n'est pas absolue. On sait, en outre, que l'encodage est à tendance impressionniste. Pour cette raison, la critique s'intéresse désormais plus aux conséquences de l'utilisation du dialecte dans l'économie de l'univers fictionnel créé, ce que Ferguson [1998] nomme ficto-linguistics qu'à l'authenticité ou à la cohérence de l'encodage. Chapman [1994:1] explique :

Variations in accent, social or regional, and in personal voice quality, are tacitly ignored unless the text requires that they be made specific. Standard spelling neutralises speech and puts the onus on the author to show any special features.

Le fonctionnement de l'encodage dialectal serait donc très proche de ce que Leech \& Short [2007] nomment la standard non fluency (hésitations, faux-départs, etc.) qui est généralement absente du dialogue fictionnel sauf quand sa présence devient signifiante. L'utilisation du 
dialecte en fiction aurait donc un rôle fonctionnel dont le but serait d'évoquer le réel sans tenter de le dupliquer. Pour Léon [1993: 34] « il s'agit [...] de dégager une valeur connotative, de créer un effet par évocation, comme le dit Bally ». Tout encodage dialectal relève donc d'une volonté de représentation. L'auteur tente de représenter, d'évoquer le réel. La question du réalisme se pose donc avec acuité. Leech \& Short [2007 : 135] commentent :

The graphological rendering of dialect is so much a matter of impressionistic convention that it is scarcely pertinent to criticise an author for lack of realism in this field.

Dans certains cas toutefois, comme celui de Joseph dans Wuthering Heights, l'encodage réalisé est tellement précis qu'il peut faire écran à l'intelligibilité ${ }^{7}$. Il semblerait que l'identité des sœurs Bells fut découverte par un habitant de Haworth ayant reconnu chez Joseph le dialecte du Yorkshire tel qui était parlé à Haworth. On sait que Charlotte tenta de modifier les passages où l'encodage d'Emily semblait trop inintelligible mais que ses tentatives n'eurent guère plus de succès et c'est généralement la version d'Emily qui est aujourd'hui reproduite dans les éditions contemporaines.

L'anglais utilise volontiers le terme de eye-dialect, que celui-ci ait trait à la gestion de la grammaire ou bien qu'il ne concerne que l'accent. Dans ce dernier cas, il serait plus précis de parler de eye-accent, mais ce terme n'est pas utilisé. On sait en outre qu'il est parfois difficile de tracer une ligne de partage claire entre accent et dialecte. La notion de eye-dialect est probablement inspirée par celle de eye-rime : il s'agit de donner l'illusion de l'utilisation d'un dialecte par l'utilisation d'une orthographe déviante alors que l'oralisation de la forme écrite renvoie à une prononciation standard. Le cas de wos est caractéristique de ce phénomène. En effet, la présence d'un $/ \mathrm{w} /$ devant un $<\mathrm{a}>$ conduit à l'arrondissement de la voyelle et donne /wpz/ (sauf si la consonne qui suit est une vélaire). La forme écrite wos est donc plus conforme à la prononciation standard. Il s'agit ici de l'illusion d'un écart. Dans le même esprit, les formes réduites sont très utilisées à l'oral. On a donc tout lieu de penser que ces formes sont aussi utilisées dans le discours direct de fiction, qui est censé représenter des paroles proférées. Or, la notation graphique de ces formes est perçue comme relevant du dialecte ou évoquant un style relâché. Thomas Wolfe [Ahmad 2007 : 283] écrit :

Even the dead will quarrel an' bicker [...].

La graphie <an'> pour and marque un écart à l'écrit alors que la forme /on/ serait, à l'oral, perçue comme tout à fait standard. La forme pleine serait fautive à l'oral.

\subsection{Réception de l'encodage}

Jusqu'au milieu du XIXe siècle, la présence de dialecte en fiction servait à situer socialement les différents personnages. Les serviteurs parlaient plus volontiers en dialecte que leurs maitres. L'opposition pouvait aussi marquer la différence entre les hommes et les femmes, les enfants et leurs parents, etc. Les marques dialectales ressurgissent en outre dans des moments de tension émotionnelle et elles sont donc, dans ce cas, souvent associées à des traits paralinguistiques vocaux qui eux aussi servent à encoder l'oralité. Si une tradition ancienne consiste à ne faire parler en dialecte que des personnages de comédie, du bas peuple ou les villains, l'évolution de la littérature rend les choses plus complexes et le positionnement narratif est déterminant dans l'évaluation. Les revendications identitaires,

\footnotetext{
${ }^{7}$ Sur ces points, voir Ferguson [1998].
} 
linguistiques, sociologiques et politiques prennent aujourd'hui souvent appui sur l'encodage dialectal.

Quelles que soient les motivations, variables selon les auteurs et les périodes littéraires, l'utilisation de traits dialectaux est motivée et est donc, dans une certaine mesure, signifiante. Black [2006 : 89] affirme :

Relevance theoreticians must assume that such use of dialect is motivated, and contributes to the meaning of an utterance through the generation of weak implicatures.

[...] We may therefore feel excluded or perhaps attracted by the strangeness.

L'argument de Black est que l'utilisation du dialecte change notre cadre de référence cognitif. Le déchiffrage correct des implicatures inhérentes à l'utilisation d'un dialecte demande un effort plus grand de la part du lecteur. Comme pour les métaphores, la bonne compréhension peut donner lieu à un sentiment d'appartenance et de communauté (empathie). La même cause peut toutefois avoir un effet inverse (distanciation). Tout dépend du type de dialecte qui est encodé, du rapport que le lecteur entretient avec ce dialecte ainsi que du degré d'encodage opéré par l'auteur. Dans la Relevance Theory, on considère qu'un acte de communication perçu comme trop obscur marque un manque de volonté véritable de communiquer. Un encodage dialectal trop opaque, que la théorie de la Politesse Linguistique qualifierait d'acte menaçant, peut être perçu pas certains lecteurs comme une violation du pacte qui unit lecteur et narrateur. On sait que certains lecteurs anglais ne parviennent pas à lire les romans de Irvine Welsh alors que d'autres s'accommodent très rapidement du code utilisé. Les implicatures créées par l'encodage dialectal et qui contribuent à l'évaluation d'un personnage sont donc analogues à ce qui se passe en interaction naturelle.

Plusieurs cadres ont été proposés pour étudier le fonctionnement de l'encodage dialectal en fiction. Crystal [2004 : 512], par exemple, propose une liste de formes utilisées pour représenter les dialectes à l'écrit. La plupart de ces formes sont aussi utilisées par Maugham :

- non accord entre le sujet et le verbe (They has)

- formes non standard de l'auxiliaire (ain't)

- participes passés non standard (took)

- gestion particulière des pronoms (hisself)

- utilisation spéciale des prépositions (a-purpose)

- $\quad$ utilisation de la double négation (isn't no more)

- adverbes particuliers

- choix lexicaux (lay /lie)

- élision de consonnes (Lor bless you)

- Eye-dialect (dunno)

Cette liste, ouverte, a le mérite d'exister mais pose problème. En effet, elle se concentre sur les conséquences lexicales ou grammaticales alors qu'il paraît plus judicieux de partir des causes qui sont avant tout graphiques. Sorrels, dans Burkett [1978 : 177-184], s'intéresse à l'encodage du dialecte rural de la Floride et propose trois catégories qui sont :

i) la variation de la prononciation.

ii) l'utilisation d'un lexique spécifique

iii) l'utilisation de formes grammaticales particulières 
Si cette présentation paraît plus synthétique, en regroupant les différents domaines (lexicologie, grammaire, phonétique), on constate que les mêmes causes produisent parfois des effets différents. Ainsi, la forme he say (qui marque une forme grammaticale dialectale) correspond à la suppression d'une lettre, tout comme doin', dans laquelle il s'agit d'une prononciation marquée. Les manipulations possibles de la graphie offertes à un auteur sont en nombre limité et se sont ces manipulations qui font du dialecte fictionnel une extension des procédés qui ont cours en langue. En d'autres termes, plutôt que de prendre les spécificités du dialecte cible comme point de départ (les effets), on prendra comme point de départ les procédés graphiques afin d'en étudier les champs d'application. Cette approche correspond à l'expérience de lecture et tente de circonscrire le travail cognitif à l'œuvre quand on lit un texte utilisant des formes dialectales. On peut ainsi réduire l'encodage dialectal à trois procédés graphiques fondamentaux, pouvant être combinés : l'insertion de graphème $(\mathrm{s})$, la suppression de graphème $(\mathrm{s})$ et la substitution de graphèmes.

\title{
3. Le Cockney de Somerset Maugham
}

Liza of Lambeth, publié en 1897 par Somerset Maugham, appartient à l'école du réalisme social et l'auteur est considéré comme un slum writer. Dans ce roman d'adultère, Maugham puise dans les connaissances que son métier de médecin lui procure pour décrire la vie des habitants du quartier de Lambeth à Londres dans les années 1850. Tous les personnages appartiennent à la working-class et tous utilisent le dialecte / accent Cockney. Maugham explique :

They expressed themselves with a cockney aptness, and I think I have reproduced their manner of speech with fair accuracy. $(L L, i x)$

La précision de l'encodage est donc revendiquée. Le fait que tous les personnages utilisent un dialecte identique, le Cockney, a pour effet de plonger le lecteur dans un univers linguistique et cognitif stable qui devient son seul repère en discours direct. Les premières pages passées, le lecteur n'éprouve que peu de difficultés à s'accommoder du code linguistique en raison de la transparence de l'encodage.

\subsection{Suppression de graphème(s)}

\section{- Lexicalisation}

Les deux premiers cas de suppression de graphèmes concernent des expressions bien connues et clairement associées au Cockney :

\author{
Bli'me if I will (5) \\ Bli'me if I know wot yer mean (89)
}

Cette expression était à l'origine: God Blind me. On remarque que la suppression de God relève de l'euphémisme (qui évite le blasphème). La graphie suggère ici l'élision $\mathrm{du} / \mathrm{d} / \mathrm{et}$ l'assimilation de /n/ en /m/. L'expression se trouve aussi sous la forme Cor / Gor blimey. Cet encodage - qui relève de la simplification de la chaîne parlée - a donné la forme blimey, attestée par les dictionnaires. Le OED donne 11 résultats pour le XXe siècle et 1 pour le XIXe. 
De même, l'expression Go' lumme! (38) qui viendrait de Lord / God Love me! a subi le même processus d'intégration : suppression de God et de la finale du verbe pour entrer dans le lexique. Contrairement à blimey toutefois, on note que la terminaison n'a pas subi d'ajout $(<y>)$.

\section{- Elision $d u<g>$ ou g-dropping}

La forme in <ing>, qu'elle tende vers le pôle nominal ou vers le pôle verbal, est presque systématiquement représentée par $<$ in' $>$ chez Maugham. Elle indique ici la perte du caractère vélaire de $/ \mathrm{y} /$.

Wot was yer doin' this afternoon, Liza? (13)

You was showin' off thet new dress of yours (13-14)

I'm not worth troublin' abaht. (18)

Somethin' ter keep me tergether (58)

And my 'ead's been simply slittin' (80)

Cette forme dialectale est très fréquente en Cockney mais aussi dans d'autres accents / dialectes de l'anglais. Il faut donc compter sur les autres traits encodés pour lui attribuer la connotation souhaitée. On sait qu'à une époque, le g-dropping était caractéristique de la $U-R P$ avec l'exemple devenu caricatural de shootin' $n$ ' huntin', sans h-dropping toutefois, contrairement au dernier exemple. L'accumulation du g-dropping (conjugué à d'autres traits) participe de l'efficacité du procédé :

Wot with the work I 'ave ter do, lookin' after you and the cookin' and gettin' everythin' ready and doin' all the 'ousework, and goin' aht charring besides (19).

Cet encodage, en soi, ne renseigne que très peu. Il n'est d'ailleurs souvent qu'un marqueur de parlure relâchée.

\section{- Elision $d u / h /$}

Le Cockney est connu pour sa tendance à ne pas prononcer le $/ \mathrm{h} /$. On trouve de nombreux exemples dans $L L$. La perte du graphème est systématiquement notée par la présence de l'apostrophe. On note une préférence pour la suppression $\mathrm{du} / \mathrm{h} /$ à l'initiale. Ce phénomène concerne les mots lexicaux :

I 'ope (4).

To the 'orspital (22)

I can't 'elp thet (21)

They won't 'urt you (57)

Yer'air (24)

If she dares ter touch a 'air of your 'ead (105)

Dans le dernier exemple, l'utilisation de l'article indéfini $a$ révèle la conscience phonologique aigue de l'auteur. En effet, s'il s'agissait d'une simple suppression du /h/, l'article indéfini serait an puisque le phonème suivant l'article indéfini serait un son voyelle. Ici, c'est un coup de glotte qui remplace le /h/. Le / $/$ / étant un phonème consonne, il n'y a pas de changement 
pour l'article. Il s'agit moins d'une perte que d'une substitution (h-switching semblerait plus approprié).

Pour les noms propres, l'utilisation systématique de la majuscule souligne une sensibilité certaine au code graphique traditionnel malgré son détournement assumé :

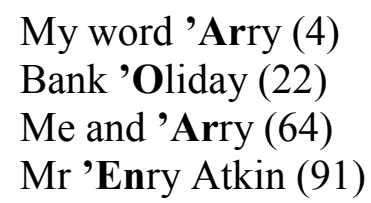

On trouve en langue, un phénomène équivalent avec le mot hostler / ostler dont la prononciation est dans les deux cas /'pslə/ avec une possibilité de prononciation orthographique en /'hpsla/ pour holster. Le nom hostler ( $<$ moyen latin hostilarius) a donné l'orthographe phonétique ostler. Traditionnellement toutefois, c'est la prononciation orthographique qui l'emporte sur l'orthographe phonétique avec des mots comme hotel, anciennement prononcé sans $/ \mathrm{h} /$, ou encore historical, prononcé avec ou sans $/ \mathrm{h} /$ quand il suit an.

A l'intérieur des mots, le phénomène est aussi présent, quoique de manière moindre en raison d'une fréquence plus faible avec: I be'aved by my mother (21) ou Well, I got yer aht, any'ow (78) qui sont à rapprocher en langue du cas de vehicle, qui accepte, en anglais américain, une prononciation orthographique avec $/ \mathrm{h} /$.

La fréquence du procédé peut être très grande à l'intérieur du même énoncé :

So 'ere to the 'ealth of Mr 'Enry Atkin's an' 'is future missus (91)

Les mots grammaticaux sont eux aussi sujets à modification :

'Oo's thet? (15)

And 'ow does your ladyship ter-day? (23)

Le cas de have est plus complexe. Pour have lexical, on trouve différents cas :

I would let 'er 'ave it (105)

if you 'ave any more I'll git a divorce, that I will (5)

La forme have to est elle aussi sujette à élision :

Wot with the work I 'ave ter do (19)

L'auxiliaire subit lui aussi une élision partielle à la forme interrogative, ce qui indique clairement un fonctionnement dialectal :

Wot 'ave you been blubberin' abaht? (98)

Wot 'ave you got yer 'air in them things for? (24)

Oh, I 'ave got sich a 'ead on me this morning (52)

La forme faible traditionnelle /ov/ serait tout à fait acceptable mais l'élision $\mathrm{du} / \mathrm{h} /$ tout en conservant sa valeur pleine à la voyelle est ici problématique. Le même procédé est utilisé à la forme affirmative. On passe donc dans le eye-dialect puisqu'une forme réduite serait utilisée à l'oral. La forme réduite de l'auxiliaire ne serait toutefois pas */æv/ mais /həv//əv/ ou /v/. A 
cet égard on doit considérer les formes clitiques comme des cas d'écriture phonétique institutionnalisées.

- Formes réduites et eye-dialect

Les exemples qui suivent nous font entrer dans le eye-dialect à proprement parler. Le cas de and transformé en an' est extrêmement fréquent :

You an' me'll dance together

I'd go an' tike yer dirty money? (20)

La conjonction and possède de nombreuses formes réduites et l'orthographe suggérée marque la chute de la dentale, facultative mais fréquente, alors qu'elle est quasi obligatoire devant une dentale (tike).

Le pronom sujet he possède lui aussi une forme faible /i/. S'il est fréquent de lire que la forme /i/ ne se rencontre que très peu en début d'énoncé, il s'agit d'une convention qui ne correspond pas à la réalité. Ainsi les formes utilisées par Maugham relèvent-elles du eyedialect :

' $\mathbf{E}$ is lite (30)

'E said 'e'd tike me to the ply! (55)

'e's not tryin' ter git 'er aht

I think 'e lives up at the other end (14)

Il en va de même pour le déterminant possessif ou le pronom personnel complément pour lesquels la forme réduite est utilisée à l'oral :

Look at 'er stockin's! (10)

E arst me ter go with 'im, but I wouldn't (24)

I've only spoke to 'im once (16)

Le pronom réfléchi himself possède lui aussi une forme faible /Im'self/ :

I'm sure 'e ought ter be ashamed of 'imself (59)

To call me to 'Imself (138)

On remarque que l'indication de l'élision $\mathrm{du} / \mathrm{h} /$ est doublée de l'utilisation de la majuscule dans le dernier exemple où il est fait référence à dieu. Comme dans le cas des noms propres, l'influence de l'écrit est évidente.

Le eye-dialect concerne aussi des items lexicaux isolés comme all right / alright ou perhaps. La supression $\mathrm{du}<\mathrm{l}>$ dans alright et de $<\mathrm{e}>$ remplacé par une apostrophe et $<\mathrm{h}>$ qui lui n'est pas remplacé par une apostrophe le confirme :

Arright, Liza (77)

P'raps 'Arry won't be there (29)

En effet, le /1/ peut être élidé dans all right / alright et on peut avoir une compression dans perhaps comme le confirment les transcriptions du LPD : /o:l'rai/ /pə'hæps/ /præps/. Le cas de governor est intéressant : 
Don't bust yerself, guv'nor! (37)

La suppression de <er $>$ marque en fait la syllabification du /n/ devant /o/. Enfin, l'exemple qui suit relève de la modification de la chaîne parlée :

S'at right, Liza? (33)

On constate la suppression de $<\mathrm{i}>$ et du graphème $<$ th $>$. Le cas de regular est plus ambigu :

It seems more reg'lar like to 'ave banns (64)

La suppression $\mathrm{du}<\mathrm{u}>$ peut laisser penser qu'il s'agit d'une réalisation du type /'reglə/ alors qu'il y a de fortes chances qu'il ne s'agisse que de la réalisation /jə/ tout à fait naturelle même si une prononciation/ju/ est possible.

Avec ces exemples, on touche aux limites de la manipulation graphique qui ne fait qu'évoquer une prononciation déviante alors qu'elle reproduit la prononciation standard plus fidèlement que la graphie traditionnelle.

\subsection{Insertion de graphème(s)}

Les cas d'insertion de graphème(s) sont bien moins nombreux. L'insertion d'un $<\mathrm{r}>$ dans 'E's gone and lorst it! (91) renvoie à la prononciation archaïque de /p/ (qui devient/o:/) que l'on rencontre encore aujourd'hui en $U-R P$. Steve Bell utilise le même procédé pour la reine censée dire gort lorst/'go:t 'lo:st/ pour got lost.

Un trait caractéristique du Cockney est l'insertion d'un /h/ là où il n'y en a pas en R.P. à des fins d'intensification. Il s'agit ici d'un marqueur d'emphase, comme le souligne Wells [1982: 322]. On rencontre plusieurs occurrences de ce phénomène dans $L L$ :

Hextra special (7)

I got the mayterials in the West Hend, didn't I? (8)

The British Hempire (123)

La conscience phonétique de Somerset Maugham dans son utilisation de ce phénomène est ici éclatante. On peut émettre l'hypothèse que la rareté de ce phénomène est due au fait qu'il n'y a pas ou peu d'équivalents en langue où l'on ajoute un graphème supplémentaire à une forme graphique pour rendre compte de sa prononciation.

\subsection{Substitution de graphèmes}

\section{- Le cas des diphtongues}

Le Cockney est connu pour ses diphtongues caractéristiques, ce que l'on nomme généralement diphthong shift et que l'on peut représenter ainsi (d'après Wells [1982 : 308]) : 


\begin{tabular}{|c|c|}
\hline R.P. & Cockney \\
\hline $\mathrm{i}:$ & $\partial \mathrm{i}$ \\
\hline eI & aI \\
\hline aI & DI \\
\hline OI & OI \\
\hline
\end{tabular}

\section{$\bullet / \mathrm{eI} / \rightarrow$ /aI/}

En R.P, la diphtongue /ai/ est souvent le résultat de $<\mathrm{i}>$ accentué suivi d'une consonne et d'une voyelle (souvent $<\mathrm{e}>$ ) ou de $<\mathrm{ai}>$. Il est donc naturel que pour évoquer la diphtongue Cockney /aI/, Maugham substitue un $<\mathrm{i}>$ au graphème d'origine.

- Dans la plupart des cas, on retrouve une relation phono-graphématique extrêmement régulière du type $<\mathrm{i}>\mathrm{C}<\mathrm{e}>\#$ : bibe (89), fice (11), gime (78), (40), nime (53), sime (59), tike (24), wiges (21), wike (81), wiste (45).

- Les mots undertikin' (138) et mistikin' (97) (take + -ING), Siving-bank (107) (save + -ING) dressmiker (8) (make + -er), peuvent être assimilés à ce fonctionnement.

- Plus rarement, on trouve $<\mathrm{i}>\mathrm{C} \mathrm{V}$ avec: lidies (40) et fital (64). Au vu de la moindre fréquence d'utilisation, on peut émettre l'hypothèse que la forme $<\mathrm{i}>\mathrm{C}<\mathrm{e}>$ étant plus aisée à reconnaître visuellement, elle est plus utilisée que la forme $<\mathrm{i}>\mathrm{C} \mathrm{V}$.

- Le cas de kite fice (47) offre une juxtaposition entre une forme graphique standard et une forme marquée sur le plan dialectal, ce qui aide le lecteur dans son processus d'accoutumance à l'encodage proposé.

- On trouve peu de cas où le digraphe $<$ ai $>$ est sujet à modification graphique :

Wite (57)

Afride (68)

Outre la substitution de $<$ ai $>$ par $<\mathrm{i}>$, un rééquilibrage orthographique est opéré. Sans l'ajout d'une $<$ e $>$ final, la substitution aurait donné /wit/ et /o'frid/. Avec l'ajout du $<$ e $>$, qui reproduit le contexte $<\mathrm{i}>\mathrm{C}<\mathrm{e}>$, le déchiffrage est facilité.

- Le digraphe <ay> en fin de morphème est régulièrement prononcé /eI/ et est directement remplacé par $<\mathrm{y}\rangle$, régulièrement prononcé /aI/ en fin de morphème, comme dans July, reply, imply, justify, etc. On trouve :

Oo, I sy (6)

I've put it awy (20)

If I did I'd py for myself an' go alone. (64)

\section{$\bullet / \mathbf{a v} / \rightarrow / \mathfrak{a} /$}

La diphtongue /av/ a tendance à être réalisée comme une monophtongue [æ:] en Cockney. $\mathrm{C}$ 'est ce que semblent indiquer les graphies suivantes $<\mathrm{ah}>$ qui indiquent un allongement :

I saw the pawn-broker dahn the road (7)

Come aht for a walk, Liza, will yer? (15)

I'm abaht done (10) 


\section{$\bullet / \mathbf{o r} / \rightarrow / \mathbf{o r} /$}

Les tentatives d'encodage graphique du passage de /or/ à /or/ sont rares :

Come an' jine in' (45)

Cette modification est fondée sur le même procédé que précédemment mais le décodage en /aI/ ne rend pas compte de la prononciation Cockney/oI/. L'auteur est aux prises avec un code graphique trop restreint pour rendre compte des nuances phonétiques. Il en a éminemment conscience et ne réitère pas la tentative.

- Cette conscience explique l'absence de tentative de rendre compte du passage de /i:/ à [əi] mais on est surpris que le passage de /ai/ à /or/ ne soit pas présent avec des formes du type toim(e) pour time ou foin(e) pour fine par exemple. La raison est sans doute la faible fréquence de cette graphie en anglais.

- Le cas de Good biz (5) pour Good Bye relève du eye-dialect. Le cas de straddlewyse (44) est peut-être plus complexe. En effet, on sait que le Cockney aurait [DI] mais l'accent populaire de Londres aurait [aI]. Intuitivement, on peut imaginer que le passage de $<\mathrm{i}>$ à $<y>$ suggère un point d'attaque de la diphtongue plus ouvert. Cette lecture reste bien sûr sujette à caution.

\section{- Inversion de certains phonèmes}

Le Cockney est connu pour sa distribution aléatoire de certains phonèmes dans certains items lexicaux, notamment /e//I/ et /e/ /æ/. Wells [1982: 304-5] donne par exemple get et catch. $L L$ en propose de nombreux exemples dans lesquels le changement de graphème suffit à évoquer le phénomène :

\section{$\bullet / \mathbf{e} / \rightarrow / \mathbf{I} /(<\mathrm{e}>\rightarrow<\mathbf{i}>)$}

I'll git a divorce, that I will (5)

togither (87)

Take care yer don't git into trouble, thet's all (89)

\section{$\bullet / \mathfrak{a} / \rightarrow / \mathbf{e} /(<\mathbf{a}>\rightarrow<\mathbf{e}>)$}

Thet's it; but wot was you doin' in there? (7)

Ketch 'old on 'er, Bill (11)

Oo's thet? (15)

$\mathrm{Na}$, not thet (20)

- La voyelle de STRUT a un point d'articulation plus antérieur en Cockney qu'en R.P. comme semblent suggérer les exemples qui suivent où le $<\mathrm{u}>$ est remplacé par $<\mathrm{e}>$ ou $<\mathrm{i}>$ :

Didn't I jest? (53)

'Ow a gentleman can use sich language, I dunno (60) 
- De même, la voyelle de NURSE a une articulation plus antérieure, comme semblent le suggérer les exemples :

Fust class (24)

Ah, my fust 'usband (59)

- Eye-dialect

La graphie <-er> dans les cas qui suivent et qui concernent to, you et ought to relève du eyedialect :

Dressed ter death, and kill the fashion (7)

'You think I'd steal it,' she went on. 'I know yer! D'yer think Well, I can't 'elp thet, can I (21)

You oughter be ashamed of yourself (107)

En effet, on sait que dans un accent non rhotique, $<$ er $>$ inaccentué se prononce généralement /a/, ce qui rend compte de la forme réduite de to + consonne $(/ \mathrm{t} ə /)$ et de la forme faible de you $(/ \mathrm{j} / /)$. Le lectorat visé a donc, dans la conscience de l'auteur, un accent non rhotique. Un Américain lisant cet encodage pourrait interpréter celui-ci comme évoquant l'ajout d'un /r/, ce qui est impossible. Si pour you et to l'auteur se contente de remplacer $<$ ou $>$ et $<0<$ par $<$ er $>$, ce procédé est accompagné d'un amalgame dans oughter. Comme pour le cas de kite fice évoqué plus haut, l'exemple qui suit joue sur la similitude entre l'orthographe traditionnelle et l'encodage :

Yesterday an' ter-day's two different things (16)

L'étymologie nous apprend toutefois que yesterday vient du vieil anglais geostran dæg dans lequel on retrouve la graphie actuelle alors que today vient du vieil anglais to dæge. Toutefois, la juxtaposition des deux formes contribue à la facilité du décodage.

Dans le même ordre d'idée, les formes suivantes relèvent elles aussi du eye-dialect:

That's wot they all says (5)

The villin! (99)

Don't bust yerself, guv'nor! (37)

I say, guv'ner (69)

La forme wot rappelle wos citée plus haut. Un $<\mathrm{a}>$ graphique précédé d'un $/ \mathrm{w} /$ prend régulièrement la valeur $/ \mathrm{p} /$. Le digraphe $<\mathrm{ai}>$ inaccentué en fin de morphème donne $/ \mathrm{I} / \mathrm{ou} / \mathrm{a} /$ (voir portrait déjà expliqué). Enfin, le passage de $<$ or $>$ à $<$ er $>$ rappelle le fonctionnement de mots comme translator vs. interpreter, tous deux réalisés /ə/. Il est remarquable que les deux formes coexistent sous la plume de Maugham.

Enfin, les exemples qui suivent sont difficilement interprétables et relèvent de la convention d'écriture dialectale plutôt que de l'encodage d'un accent particulier.

I never thought of arskin' yer, Liza (22)

Tom never arst me! (61) 
I ain't got the chanst any more (29)

\section{Conclusion}

La tension qui existe entre prononciation et graphie a laissé son empreinte dans la langue anglaise depuis ses origines. Le Grand Changement Vocalique est à juste titre souvent évoqué pour expliquer les écarts qui existent aujourd'hui. L'argument défendu ici est que l'écriture phonétique possède un fonctionnement comparable en langue et dans l'encodage dialectal. La preuve en est que quand un encodage d'origine dialectal est suffisamment repris, il intègre le lexique et son orthographe s'institutionnalise (wanna, Blimey, etc.).

Le texte pris comme corpus nous apprend plusieurs choses. Les trois procédés graphiques répertoriés sont i) la suppression de graphème(s); ii) l'ajout de graphème(s); iii) la substitution de graphèmes avec la possibilité de combiner ces trois manipulations. Par ailleurs, le nombre de traits encodés pour évoquer le Cockney est relativement faible (perte du $/ \mathrm{h} /$, insertion $\mathrm{du} / \mathrm{h} /$, représentation des diphtongues spécifiques et quelques manipulations graphiques pour évoquer des réalisations phonémiques particulières). Toutefois, le texte de Somerset Maugham est très dense en termes de manipulations graphiques. La plupart concernent toutefois le eye-dialect qui, parce qu'il évoque l'oralité de manière plus juste que l'orthographe traditionnelle, ne pose pas de problème de déchiffrement au lecteur. Wells [1982 : 333] souligne que Dickens n'essaye pas d'encoder les diphtongues du Cockney ou la glottalisation. G.B. Shaw, au contraire, donne une image plus précise avec en plus des diphtongues et de la glottalisation, des tentatives pour évoquer la vocalisation $\mathrm{du} / \mathrm{l} /$.

L'idée développée par Black prend ici toute sa force. Le lecteur est incité par le contexte et sa connaissance encyclopédique de l'univers Cockney à interpréter tout encodage comme une évocation de traits phonétiques propres alors qu'en réalité, ceux-ci sont peu nombreux. Quand toutefois des traits spécifiquement Cockney sont encodés (fice pour face), cet encodage est fondé sur des règles grapho-phonématiques extrêmement régulières qui rendent le décodage aisé. Plus les règles grapho-phonématiques sont complexes, moins l'encodage dialectal les utilise. Le lecteur a donc l'impression de fournir un effort très important (en raison du nombre considérable de déviations orthographiques) alors que le travail est extrêmement facilité par le fait que l'encodage s'appuie sur un fonctionnement régulier de la langue.

La présence d'expressions (Go' lumme, swop me dicky, cheese it et dans une moindre mesure bloomin') incitent le lecteur à interpréter comme relevant du Cockney tout ce qui est graphiquement déviant. Dans le roman, l'auteur souligne lui-même la difficulté de l'encodage dialectal :

Her language was emphatic, her epithets picturesque, but too forcible for reproduction. (20)

Cet appel à la coopération du lecteur, s'il révèle les limites du procédé, résume et condense le principe central à toute tentative d'encodage : le lecteur doit être prêt à compenser les insuffisances de la graphie. Sans cette volonté qui relève à la fois du willing suspension of disbelief et du principe de coopération gricéen, toute tentative reste vouée à l'échec. 


\section{Bibliographie}

AHMAD Dohra (ed.), Rotten English, New York, Norton, 2007.

AleXANDER Lynn, "Sociological Stylistics: the Politics of Dialect in Early NineteenthCentury British Fiction", South Central Modern Language Association, 1-3 November 2008, TN (unpublished).

BAUER Laurie, English Word-formation, Cambridge, Cambridge University Press, 1983.

BELL Steve, http://www.guardian.co.uk/

BLACK Elizabeth, Pragmatic Stylistics, Edinburgh, Edinburgh University Press, 2006.

BurKeTt Eva, American English Dialects in Literature. Metuchen, The Scarecrow Press, 1978.

CHAPMAN Raymond, The Treatment of Sounds in Language and Literature, London: Blackwell, 1984.

---, Forms of Speech in Victorian Fiction, London, Longman, 1994.

COOPER Andrew, “'Folk-speech' and 'book English': Re-presentation of Dialect in Hardy's Novels", Language \& Literature, vol. 3, number 1, 1994 : 21-41.

Crystal David, The Stories of English, London, Penguin, 2004.

DehaENe Stanislas, Les Neurones de la lecture, Paris, Odile Jacob, 2007.

DURAND Jacques, «La phonétique classique : 1'Association Phonétique Internationale et son alphabet », in Nguyen N., WauQuier-Gravelines S., Durand J. (eds.), Phonologie et phonétique : forme et substance, Paris, Hermès, $2005: 25-59$.

Ferguson Susan, "Drawing the Fictional Lines: Dialect and Narrative in the Victorian Novel”, Style, Spring, 1998: 245-258.

Jones Gavin, Strange Talk - The Politics of Dialect Literature in Gilded Age America, Berkeley, University of California Press, 1999.

LEECH Geoffrey \& SHORT Michael, Style in Fiction, London, Longman, 2007 (second edition).

MARTIN Jean-Pierre, La Bande sonore, Paris, José Corti, 1998.

MCINTYRE Dan, History of English, London, Routledge, 2009.

Page Norman, Speech in the English Novel, London, Longman, 1973.

SAUSSURE Ferdinand (de), Cours de linguistique générale (1916), Paris, Payot, 1972.

STEWART Garrett, Reading Voices, Literature and the Phonotext, Berkeley, University of California Press, 1990.

StOCKWELL Peter, Sociolinguistics (2003), London, Routledge, 2007 ( $2^{\text {nd }}$ edition).

Toolan Michael, "Compromising Positions: Systemic Linguistics and the Locally Managed Semiotics of Dialogue", in Davis Birch \& Maichael O'Toole (Eds), Functions of Style, London, Pinter Publishers, 1988 : 249-262.

---, "The Significations of Representing Dialect in Writing", Language \& Literature, vol. 1, number 1, 1992 : 29-46.

TourniER Jean, Précis de lexicologie anglaise, Paris, Nathan, 1994.

Webster Noah, The American Spelling Book (1783), New York, Webster Publishing Company, 1932.

Wells J.C., "British English Pronunciation Preferences: a Changing Scene", Journal of the International Phonetic Association, vol. 29, nb. 1 : 33-50. 